\title{
Capacity Optimization in Dynamically Routing Computer Network Systems
}

\author{
Nuşin Uncu*, Rızvan Erol
}

\begin{abstract}
A computer network system is a complex system with a great number of dynamic components. There are many devices in the system, such as computers, routers, lines, hubs, and switches. In addition to these hardware systems, many protocols are integrated to set the rules and provide the way of communication. Due to the nature of the system, it is hard to formulate and solve problems analytically without making any assumptions. One of the prominent problems that occur in computer systems is the line capacity assignment problem. In the previous mathematical models, message routes were predetermined and the dynamic nature of the system was neglected. This study deals with the line capacity assignment problem under a dynamically routing policy. Four different computer network topologies are used and solved by two heuristic algorithms via simulation. A dynamic search approach based on the occupancy rate of lines is used to define the consecutive routes of messages. The performances of harmony search and genetic algorithms via simulation are compared with the results of OptQuest, one of the optimization packet programs embedded in simulation software Arena ${ }^{\circledR}$.
\end{abstract}

Keywords: computer networks; harmony search; genetic algorithms; optimization via simulation

\section{INTRODUCTION}

Studies on computer network systems date back to the 1970s. The common goal is to minimize the cost of the system while reducing congestion and latency in a perfectly designed network system under well-defined routing policies. Computer network optimization problems range from the physical structure of network to the optimization of parameters in the network. They can be classified under three categories:

1) Topology optimization or network design problems

2) Routing problems

3) Line capacity and resource allocation problems.

A minimum cost topology design is given in [1]. Topology optimization and network design problems have been solved with search algorithms, relaxation methods and evolutionary algorithms [2-5]

The routing problem is formulated in [6] as a nonlinear multi-product flow problem and solved with the decomposition method. ARPA, which is a wide area packet switching network, and NASDAQ as a distributed network structure are used in [7]. They investigated the location, capacity determination, routing and reliability problems of specific connections for each structure. They proposed queuing theory, pure approach, functional simulation approach, Moore-Shannon approach and iterative heuristic approaches to solve these problems and highlighted the shortcomings in the literature for possible studies. Another network routing problem is solved in [8] using Lagrange relaxation and subgrade optimization methods minimizing the transmission times using four different topologies. The problem of multi-packet routing was addressed in the $n \times n$ mesh topology with the rule of sending wormhole by dividing the packets into their link capacity in [9]. The primary and secondary routing problem in backbone network structures was formulated in [10]. The problem of determining the primary routes as well as the secondary routes in case of traffic density occurring in the backbone network structure was discussed in [11]. They solved the nonlinear integer mathematical model based on minimization of waiting times of messages in the system with a heuristic algorithm based on Lagrange relaxation and compared the results with the ones using greedy and Dijkstra's algorithms. Two routing policies were introduced in [12]: fixed routing, where once routes are set up they are always followed by the messages, or adaptive routing, where the routes are defined as a function of the information available when the messages arrive.

The constrained routing optimization problem in network systems was solved by particle swarm optimization algorithm in [13]. The optimum path strategy was suggested in [14] for maximum node density probability (betweeness) minimization to prevent congestion in complex networks. A FuzzyAntNet algorithm was developed in [15] using fuzzy logic and AntNet algorithm together. They compared the simulation results with the Destination Sequenced Distance Vector and AntNet algorithms. A novel routing algorithm that integrates static and dynamic routing policies using virtual clustering and flow divergence algorithms was introduced in [16]. Two traffic congestion control techniques were proposed in [17] for flow assignment and packet scheduling, for effective routing in computer networks. Dynamic routing and static routing policies were compared in [18] and emphasized that dynamic routing algorithms give better results in terms of system performance if they work fast, but any miscalculation in the routing table may increase the waiting time in the system.

The line capacity problem in packet switching computer networks was first introduced in [19]. The problem of line capacity and flow assignment in computer networks was modelled as $\mathrm{m}-\mathrm{M} / \mathrm{M} / 1$ queuing system in [20]. They formulated the objective function according to the multiproduct flow network and solved it by flow deviation method. Towards 1990s, the problem of capacity and route determination has begun to be combined into a mathematical model. The first study in this area is the work in [21]. They formulated a nonlinear integer model determining the routes and connection capacities that will minimize the cost in computer networks, where network topologies and candidate 
route sets for each node pair in communication were given, and solved it using Lagrange approximation. Resource allocation problem was considered in [22] on time-varying directed communication networks using distributed optimization method. In [23], line capacity and flow assignment problem was solved by bender decomposition method. The cost of installation was based on quality of service on different topologies and fixed capacities. A new genetic algorithm approach was developed in [24] for network line capacities and routing problem and better results than previous studies with parallel computing technique were obtained. A heuristic was proposed for continuous capacity and flow assignment problem in [25]. In the context of queuing theory, a mathematical model dealing with capacity assignment in computer networks was given in [26]. Predicting the link availability in computer network systems, especially on mobile ad-hoc has gained importance. The link availability was predicted by [27] in Mobile Ad hoc Network (MANET) considering the capacity optimization. A new topological system called as MECPerf enabling online collection of network measurements was proposed in [28]. The proposed architecture is useful on edge computing domain for online network optimization to increase system performance.

Besides the most dedicated problems on computer networks, some miscellaneous problems are noticed in the area of communication network such as path planning, resource utilization, risk analysis and reliability [29-33].

In large-scale computer communication networks (e.g. the nowadays Internet), the assignment of link capacities and selection of routes (or the assignment of flows) are extremely complex network optimization problems [23]. Even when there is no uncertainty, optimization can be very difficult if the number of design variables is large, the problem contains a diverse collection of design variable types, and little is known about the structure of the performance function [34] . The optimization via simulation methods (OvS) are used to overcome the difficulties in optimization of large scale complex systems. The related problems occur in real world systems such as health systems, telecommunications, supply chain networks, computer network systems, manufacturing systems, etc. OvS were classified in [35] according to the problem characteristics such as shape of the response surface (global as compared to local optimization), objective functions (single or multiple objectives) and parameter spaces (discrete or continuous parameters). Because of the dynamics and the complexity of the computer network systems, the method applied in this study is the OvS technique that enables to get the expected value of functions considering dynamic behaviour of the system. The part of the evaluation and generation of solutions are under considered in optimization phase. Each candidate solution is obtained by simulation then feasible solutions are evaluated and improved by optimization methods.

In this study, the capacity assignment problem in computer networks is considered with dynamically routing policies. As a contribution to the existing literature, two OvS methods are suggested to figure out the applicability and effectiveness of these methods for computer network systems.

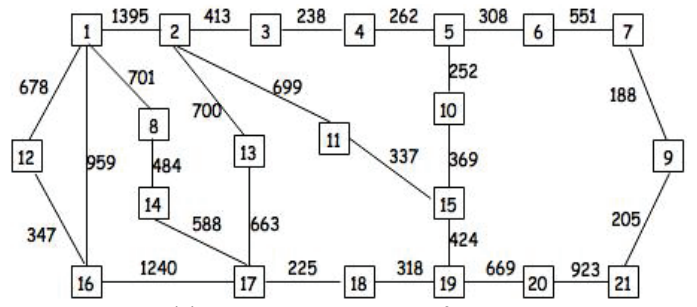

(a) The network topology of ARPA

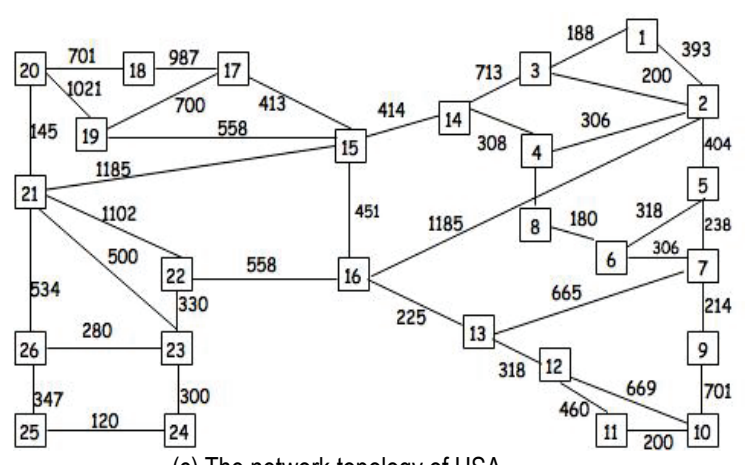

(c) The network topology of USA

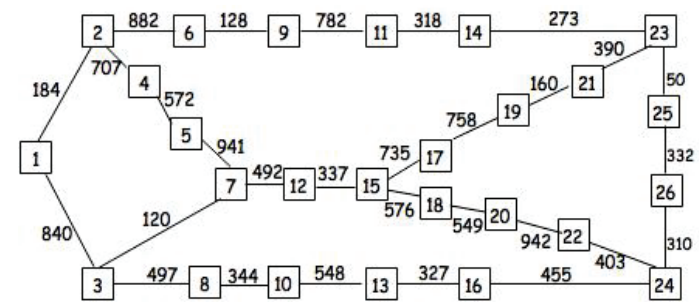

(b) The network topology of OCT
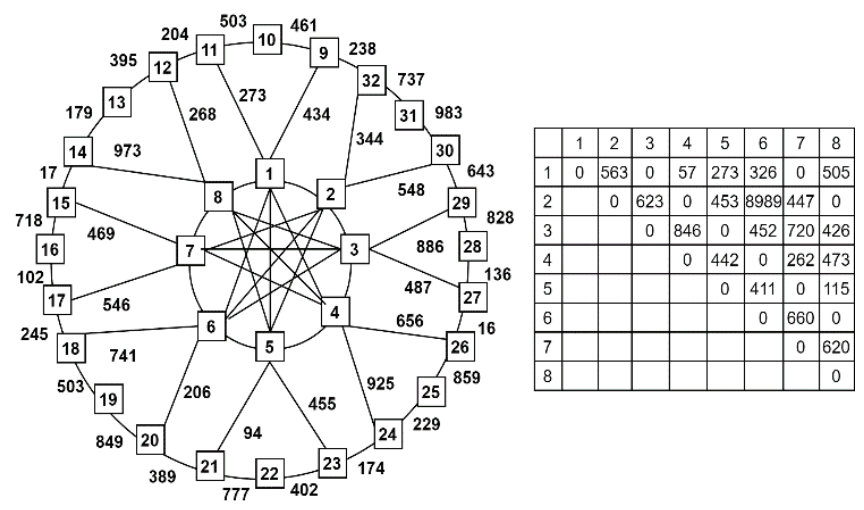

(d) The network topology of RING

Figure 1 Computer network topologies

\section{PROBLEM DEFINITION}

A computer communication network can be described as a connected and undirected graph $G=(N, E)$, where $N$ is the set of nodes and $E$ is the set of lines. Each node is considered to act as a store-and-forward switching station with infinite storage capacity, and each line is considered to be an errorfree communication channel or link. 
Table 1 The number of nodes and links for each type of topologies

\begin{tabular}{|l|c|c|c|c|}
\hline & \multicolumn{4}{|c|}{ Topologies } \\
\hline & ARPA & OCT & USA & RING \\
\hline Number of nodes & 21 & 26 & 26 & 32 \\
\hline Number of links & 26 & 29 & 41 & 60 \\
\hline
\end{tabular}

Table 2 Link capacities and costs

\begin{tabular}{|c|c|c|c|c|}
\hline $\begin{array}{c}\text { Types of } \\
\text { links }\end{array}$ & $\begin{array}{c}\text { Capacities } \\
(\mathrm{bit} / \mathrm{sec})\end{array}$ & $\begin{array}{c}\text { Setup Cost } \\
(\$ / \text { month })\end{array}$ & $\begin{array}{c}\text { Distance Cost } \\
(\$ / \text { month/mile })\end{array}$ & $\begin{array}{c}\text { Variable Cost } \\
(\$ / \text { month/bps })\end{array}$ \\
\hline 1 & 4800 & 650 & 0.4 & 0.360 \\
\hline 2 & 9600 & 750 & 0.5 & 0.252 \\
\hline 3 & 19200 & 850 & 2.1 & 0.126 \\
\hline 4 & 50000 & 850 & 4.2 & 0.030 \\
\hline 5 & 108000 & 2400 & 4.2 & 0.024 \\
\hline 6 & 230000 & 1300 & 21.0 & 0.020 \\
\hline 7 & 460000 & 1300 & 60.0 & 0.017 \\
\hline
\end{tabular}

The topologies and distances between links are taken from [36] are given in Fig. 1. In order to avoid figure complexity, the distances between nodes 1 to 8 in RING topology is represented on the right side of RING network.
Number of nodes and links for each topology is summarized in Tab. 1. Line capacities and costs for each type of line is taken from [36] and given in Tab. 2. The unit delay cost for all the topologies is $\$ 20$ per sec.

\section{OPTIMIZATION VIA SIMULATION}

The concept of using simulation and optimization methods simultaneously has been widely studied since 1970s. Although the performance of the methods differs from case to case, the general idea of the application is to find the optimal value of a function which depends on a parameter that is changed by optimization and evaluated by simulation. Starting with the conceptual model of the problem, general OvS steps followed for each metaheuristics in this study are shown in Fig. 2.

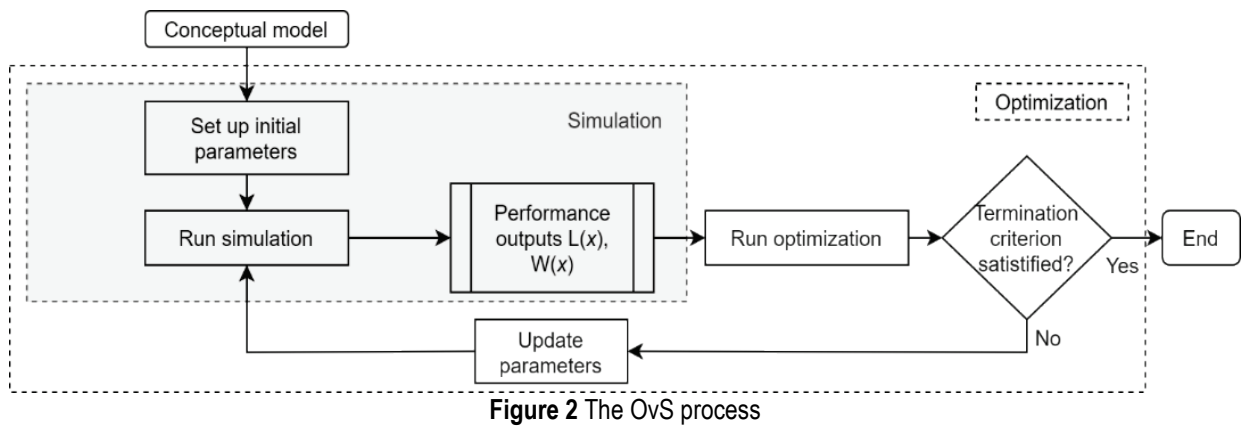

\subsection{Computer Network Simulation Optimization Model}

Computer network system can be illustrated basically in Fig. 3. In this study, the nodes shows routers and the links between these nodes are cables. There are routing protocols embedded to the nodes and there may occur queues in front of the router based on the online usage of the corresponding line.

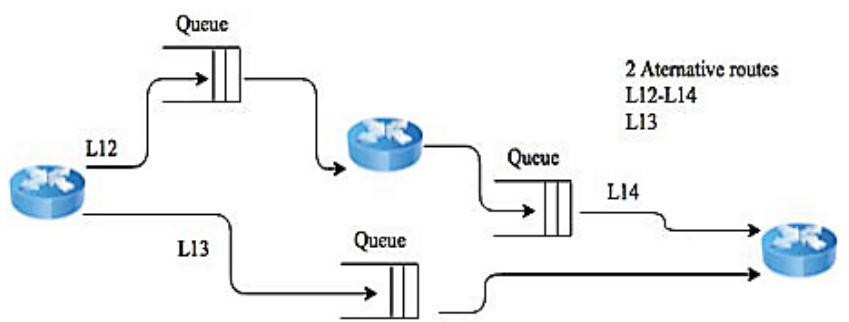

Figure 3 An exhibition of queuing model for each node

In the suggested model, messages occur on any node and destination is randomly assigned to each message. The occurrence rate of messages is exponentially distributed and inter arrival time is 4 seconds. The message visits the nodes dynamically throughout the system depending upon the links' online occupancy rate until it reaches to its destination node. So, the message finds its best route throughout the online progress. The time spend at nodes is ignored, while the delays are considered due to a congestion on the links.

If the messages do not reach its destination node in a reasonable amount of time or hop number, the message is classified as failed.

Other assumptions are explained below.

- Messages spend time relatively to the capacity of lines they visited.

- Length of messages are assigned equally likely as the sizes of 200, 300, 400, 500 and $600 \mathrm{MB}$.

- The capacity of the lines declines throughout the occupancy rate.

- The destination nodes are not predefined and have equal chance to be selected.

The simulation model includes events such as receiving the message, message enter to the first route, and subsequent events of travel on network lines based on dynamically searching algorithm of line availability, end with reaching target message node and message dispose from the network system. Through entire model the message are given attributes and variables that are used to find model statistics. An instance of simulation model and main blocks are given in Fig. 4 with a superficial flow of message due to the difficulties to screen out all the blocks in model. 


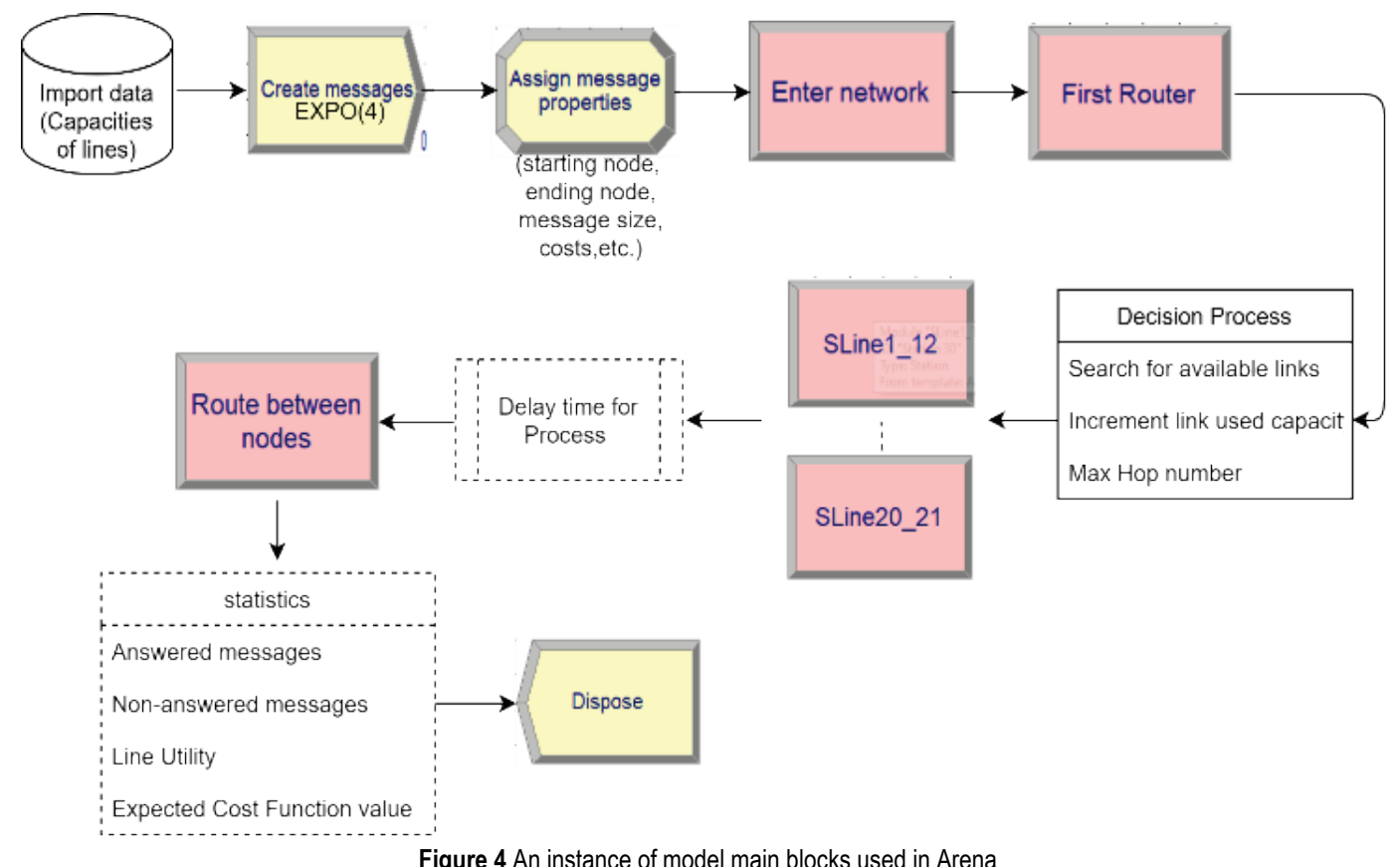

3.

The notations used in the equations are explained in Tab.

Table 3 The notation

\begin{tabular}{|l|}
\hline Decision Variable \\
$y_{i j}=\left\{\begin{array}{l|}1 \\
0\end{array}\right.$ type line(capacity) $j$ is used by link i otherwise \\
$i=1, \ldots, n, j=1, \ldots, k$
\end{tabular}

Objective function in Eq. (1) is the minimization of the total cost while assigning the optimal link capacities.

$\min z=$

$=\sum_{i \in I, j \in J}\left(s_{j} y_{i j}+d_{j} m_{i}\right)+\sum_{i \in I, j \in J} v_{j} y_{i j} L_{i}(x)+\sum_{i \in I, j \in J} b W_{i}(x)^{(1)}$

In Eq. (1), the first sum is for set up cost and cost of lines used for each link, the second is operational cost depending on the size of the messages and assigned line capacity and the third is delay cost. The operational and delay costs are expected costs and obtained by the simulation. These functions depend on the size of messages that travelled through the network as the system proceeds. Eq. (2) restricts each node to be connected just one type of line. Eq. (3) restricts the decision variables to be binary.

$$
\begin{aligned}
& \sum_{j \in J} y_{i j}=1 \quad \forall i \in I \\
& y_{i j} \in\{0,1\} \quad \forall i \in I, \forall j \in J
\end{aligned}
$$

The travel times of the messages change according to the capacity of the link and size of the message. The related formula is given in Eq. (4). It is mentioned that the time that a message reaches to its destination node increases as the utility of the link increases. The online occupancy rate is given in Eq. (5). The online occupancy rate provides the decision of route selection in the dynamically changing states of computer network system.

$\frac{1}{\mu}=\frac{\text { Message length } / \text { Link capacity }}{(1-\text { Online occupancy rate })}$

Online Occupancy Rate $=\frac{\text { Online Usage }}{\text { Total Capacity }}$

\subsubsection{Harmony Search Algorithm via Simulation}

The harmony search algorithm is one of the challenging metaheuristics presented in [37]. It is inspired from the idea of harmony of instruments. Harmony search algorithm has two parameters, harmony memory size (HMS) and harmony memory consideration rate (HMCR). HMS is the number of candidate solutions in the population. HMCR is the probability of selecting a member in HMS. In this study, an extended version, intelligent tuned harmony search algorithm, which is proposed in [38] is applied and the integration with simulation is depicted in Fig. 5. 


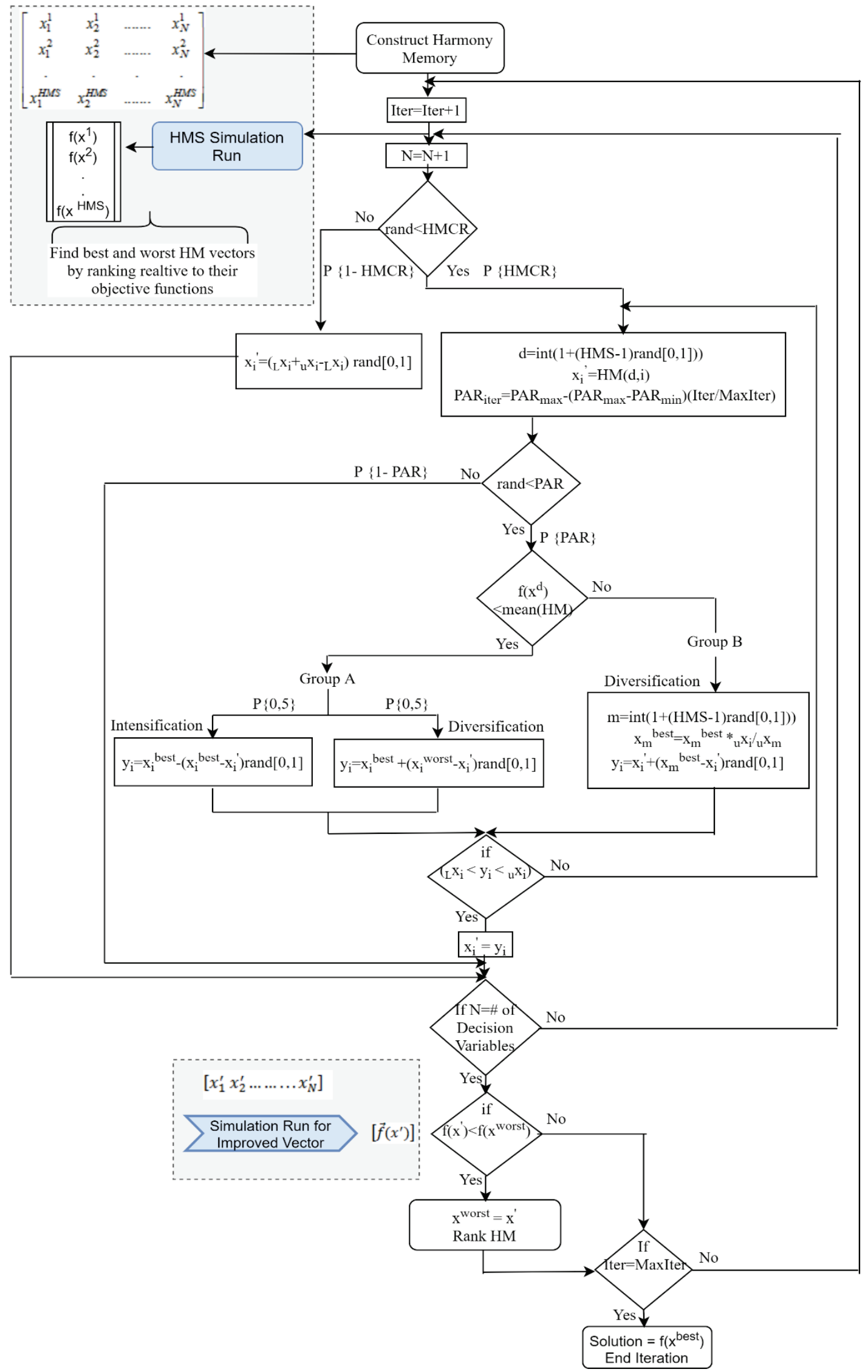

Figure 5 Intelligent tuned harmony search algorithm via simulation 
The grouping approach in intelligent tuned harmony search algorithm enables the heuristic to provide the opportunity of selecting a new member from sub-population. If the function value of selected member is less than the average function value of $H M S$, that member is classified in the group of A, otherwise in the group of B. For the members in group A, the chance of intensification or diversification is applied by a probability of 50 percent. For the members in the group B, only diversification is applied. Intelligent tuned harmony search algorithm has a new parameter, dynamic pitch adjustment rate $(P A R)$, which provides a deep and detailed search.

Each member in the population has a cost function which is estimated by simulation. Then the members are sorted by ascending order according to the value of their cost functions. An update of the harmony memory is done based on HMCR or PAR. The probability of updating any member with choosing a member in harmony memory is HMCR, while randomly change is $(1-H M C R)$. Then, another adjustment can be made by a probability of $P A R_{\text {iter }}$ or no adjustment by $\left(1-P A R_{\text {iter }}\right)$. It is calculated in Eq. (7).

$\sum_{j \in J} y_{i j}=1 \quad \forall i \in I$

$P A R_{\text {iter }}=P A R_{\max }-\left(P A R_{\max }-P A R_{\min }\right)\left(\frac{\text { current }_{\text {iter }}}{\text { max }_{\text {iter }}}\right)$

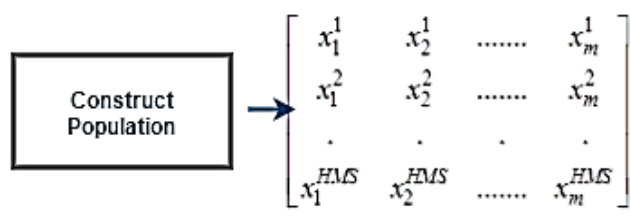

Figure 6 Genetic algorithm via simulation

Table 5 GenSimOpt with different parameters

\begin{tabular}{|c|c|c|c|c|}
\hline \multirow{2}{*}{ \# of Iterations } & \multicolumn{4}{|c|}{ Crossover Rate } \\
\cline { 2 - 5 } & 0,50 & 0,75 & 0,90 & CPU Time \\
\hline 30 & 122,84 & 118,30 & 120,74 & $4^{\prime}$ \\
\hline 50 & 118,67 & 118,30 & 120,21 & $6^{\prime} 30^{\prime \prime}$ \\
\hline 100 & 117,54 & 117,06 & 120,19 & $11^{\prime} 44^{\prime \prime}$ \\
\hline 150 & 116,92 & 109,45 & 119,23 & $1^{\prime} 47^{\prime \prime}$ \\
\hline
\end{tabular}

After some trial, the appropriate values of the harmony parameters are chosen with a $H M S$ of 150 and a $H M C R$ of 0.90 as depicted in Tab. 4.

\begin{tabular}{|c|c|c|c|c|c|}
\hline \multirow{2}{*}{ HMS } & \multirow{2}{*}{ \# of Iterations } & \multicolumn{4}{|c|}{ HMCR } \\
\hline & & 0,10 & 0,50 & 0,90 & CPU Time \\
\hline \multirow{4}{*}{20} & 30 & 141,17 & 141,17 & 143,74 & $35 "$ \\
\hline & 50 & 139,34 & 141,17 & 141,17 & $46 "$ \\
\hline & 100 & 139,11 & 139,77 & 141,17 & $1 ' 20 "$ \\
\hline & 200 & 139,11 & 139,77 & 141,17 & 2'26" \\
\hline \multirow{4}{*}{50} & 30 & 129,51 & 129,51 & 129,51 & $35^{\prime \prime}$ \\
\hline & 50 & 129,51 & 129,51 & 128,74 & $46 "$ \\
\hline & 100 & 129,51 & 129,51 & 128,52 & $1 ' 20 "$ \\
\hline & 200 & 129,51 & 129,51 & 127,78 & 2'26" \\
\hline \multirow{4}{*}{150} & 30 & 128,70 & 128,70 & 128,70 & $35^{\prime \prime}$ \\
\hline & 50 & 128,70 & 128,70 & 128,70 & $46^{\prime \prime}$ \\
\hline & 100 & 128,70 & 128,70 & 128,70 & 1'20" \\
\hline & 200 & 128,70 & 128,70 & 127,71 & $2 ' 26^{\prime \prime}$ \\
\hline
\end{tabular}

\subsubsection{Genetic Algorithm via Simulation}

Genetic algorithm was first introduced in [39] to solve some common mathematical problems. Two important operators are crossover and mutation. One way crossover is considered and ranking selection method is used to select the members that are exposed to genetic algorithm operators. Mutation rate is set to 0.01 and population size is 20 . Genetic algorithm via simulation steps are given in Fig. 6 .

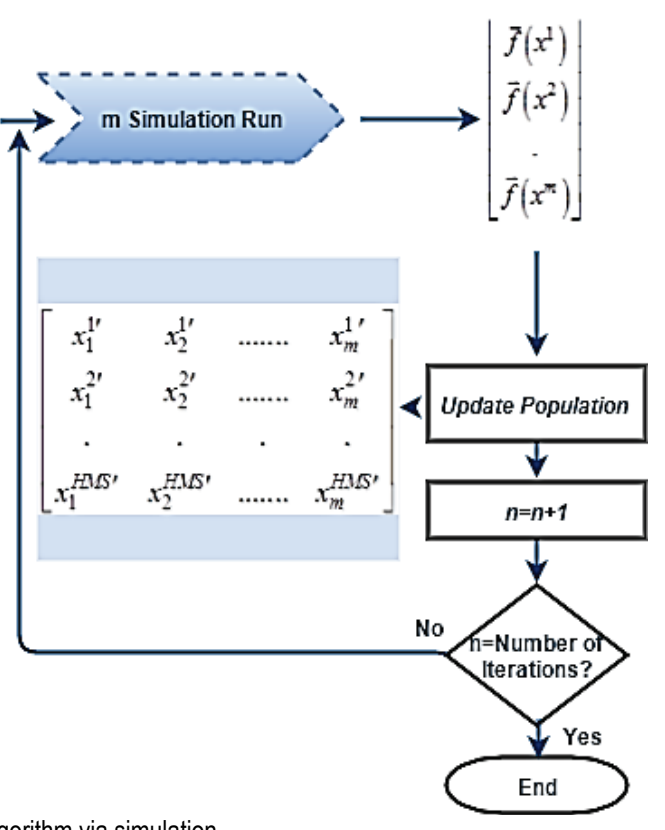

Genetic algorithm via simulation is run for different crossover rates and iterations. The optimal costs per cases and CPU times are given in Tab. 5. It is seen that with a crossover rate of 0.75 and 150 iterations, algorithm provides desirable result. 


\subsubsection{OptQuest}

OptQuest is an optimization tool embedded in simulation software Arena ${ }^{\circledR}$. Optimization algorithms in OptQuest are not explicitly known, but it is known as the combination of scatter search, advanced taboo search, linear programming, integer programming, neural networks and linear regression. OptQuest allow users to find a feasible solution for a mathematical model that imports the parameter values from simulation runs simultaneously.

\section{COMPUTATIONAL RESULTS}

Delphi $^{\mathrm{TM}} 7.0$ is used to write the algorithms. Simulation tool Arena ${ }^{\circledR}$ is embedded inside the user interface module in order to execute the OvS methods simultaneously. The harmony search and genetic algorithms are population based algorithms. The advantage of using harmony search is that it needs fewer configurations than genetic algorithms, but has a disadvantage because; only one of the candidate solutions can be renewed per each population generation. While using genetic algorithms, a group of population is updated proportionally to crossover and mutation rates. So, the process of converging optimum in simulation optimization progress is longer in time in harmony search algorithm than in genetic algorithms as seen in Fig. 7. The number of simulation runs are different. But, actually the number of population members updated for each algorithm is the same. The convergence to the optimum in genetic algorithm seems better than harmony search algorithm. The optimal is reached after 120 runs in genetic algorithms while 300 runs in harmony search. Due to the connection structures of nodes in the topologies, the performances of the algorithms result in differ. For example, genetic algorithms converges to optimal with a less number of iterations for USA topology and greater number of iterations for OCT topology among others. However, harmony search algorithm converges to optimal with a less number of iterations for ARPA topology among others.

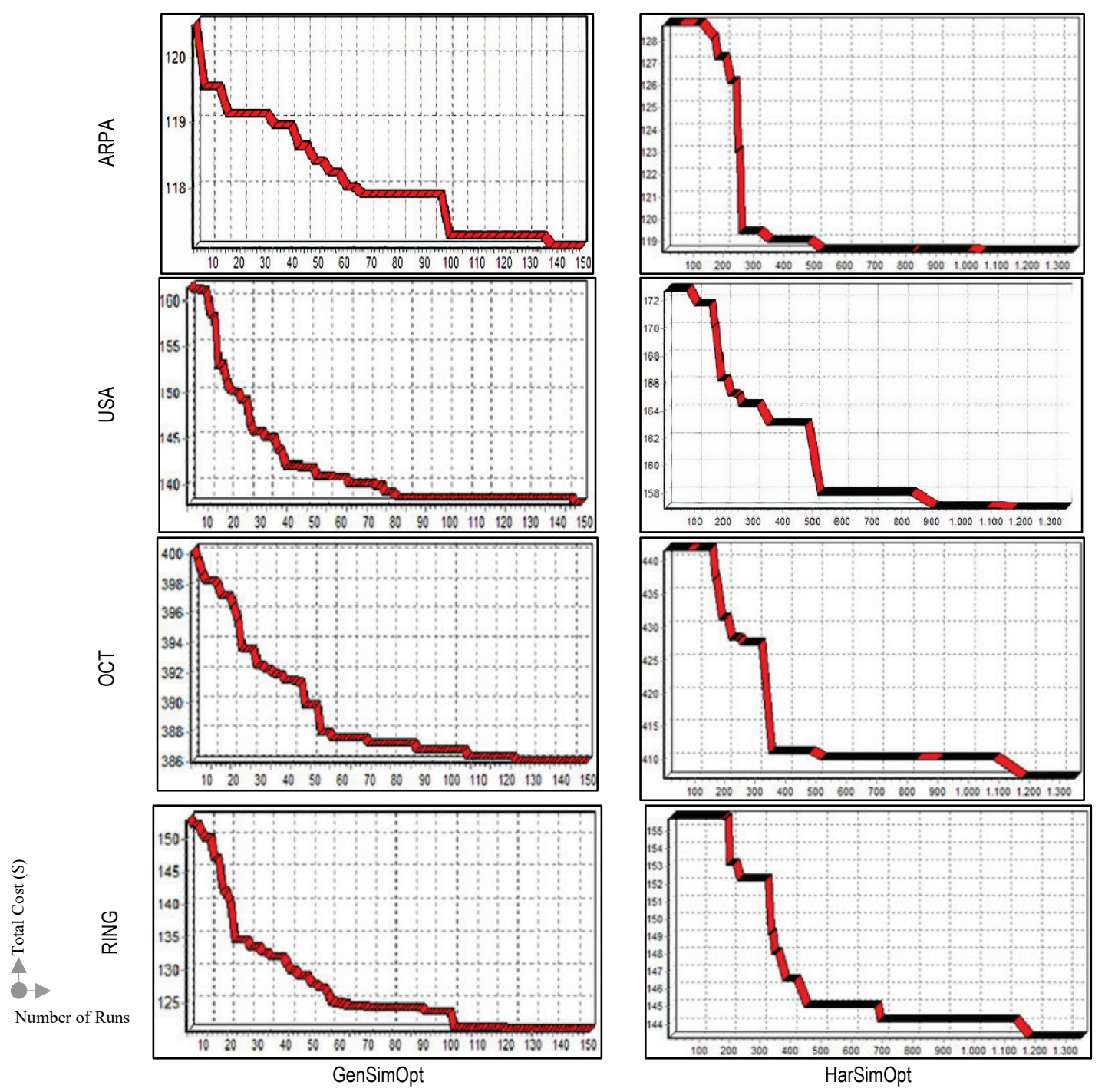

Figure 7 Improvement in total cost through number of simulation runs for each metaheuristics and topology 
OptQuest is reached a desirable optimal value after 2000 simulation runs. Fixed, variable, delay and total costs for each network topology obtained using three methods are given in Tab. 6. Based on the costs of the network system, GenSimOpt gives the minimum total cost for each topology, while the HarSimopt and OptQuest performances of converging to minimum cost change for different topologies of networks.

Table 6 Optimal costs per topology obtained by three OvS methods

\begin{tabular}{|c|c|c|c|c|c|}
\hline & Methods & $\begin{array}{c}\text { Fixed } \\
\text { Cost (\$) }\end{array}$ & $\begin{array}{c}\text { Variable } \\
\text { Cost (\$) }\end{array}$ & $\begin{array}{c}\text { Delay } \\
\text { Cost (\$) }\end{array}$ & $\begin{array}{c}\text { Total } \\
\text { Cost (\$) }\end{array}$ \\
\hline \multirow{4}{*}{ ARPA } & HarSimOpt & 72,63 & 33,31 & 12,57 & 118,51 \\
\cline { 2 - 6 } & GenSimOpt & 70,77 & 29,47 & 9,21 & 109,45 \\
\cline { 2 - 6 } & OptQuest & 74,09 & 32,34 & 13,67 & 120,10 \\
\hline \multirow{3}{*}{ OCT } & HarSimOpt & 90,88 & 42,54 & 23,46 & 156,88 \\
\cline { 2 - 6 } & GenSimOpt & 85,23 & 30,49 & 22,01 & 137,73 \\
\cline { 2 - 6 } & OptQuest & 91,6 & 24,39 & 23,9 & 139,89 \\
\hline \multirow{3}{*}{ USA } & HarSimOpt & 105,11 & 25,17 & 266,61 & 396,89 \\
\cline { 2 - 6 } & GenSimOpt & 96,43 & 21,67 & 267,83 & 385,93 \\
\cline { 2 - 6 } RING & OptQuest & 112,55 & 16,92 & 266,79 & 396,26 \\
\hline & HarSimOpt & 95,43 & 20,38 & 25,27 & 143,20 \\
\cline { 2 - 6 } & GenSimOpt & 82,28 & 17,2 & 17,6 & 120,22 \\
\cline { 2 - 6 } & OptQuest & 92,91 & 12,59 & 18,19 & 124,81 \\
\hline
\end{tabular}

The results are illustrated in Fig. 8. The USA topology have a higher delay and total cost because of the messages wait in the system to reach its destination node up to the maximum number of hops. The USA topology has two bottleneck nodes 15 and 16 that are only two nodes placed on the connections of other nodes. Once looking at the topologies one can conclude that the RING network would have been a higher cost, but the mesh structure of RING gives messages opportunities to reach its destination node via different alternative ways.

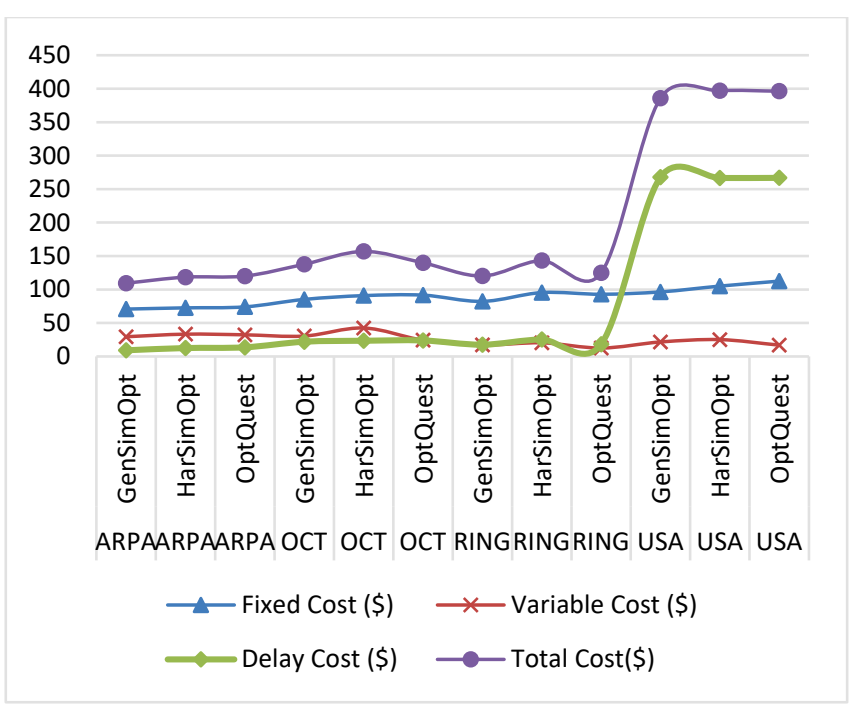

Figure 8 The results of each method for each network topology

\section{CONCLUSION}

The capacity assignment problem of computer network systems under dynamic routing conditions is solved by OvS methods involving heuristic algorithms in this study. Since there is no remarkable study for discrete event simulation
(DES) of computer network systems in the literature, the main contribution of this paper is providing the DES model of such a complex system. As another contribution, the costs are estimated not under restricted, fixed, and finite combinations based on the end-to-end routing of messages as handled in the previous studies. The simulation and optimization methods are used simultaneously with high consideration of steady-state conditions to increase the precision level. The results show that genetic algorithm gives the best results for nearly the same CPU time compared with other methods. In addition, although OptQuest is used by several researchers, its performance is not highly appreciated in computer network systems simulation optimization problem.

In this study, it is observed that due to the great amount of CPU time of simulation runs, it would be less time consuming at least the optimization method converging to the optimum by a small number of iteration. Otherwise, the OvS method becomes less efficient to solve complex optimization problems. Each heuristic used in this study is a populationbased algorithm. Although they are very efficient for solving general optimization problems, the trade-off is important among the simplicity of the algorithm, applicability, and success of convergence to optimum in a reasonable time. It can be concluded that harmony algorithm is better than genetic algorithms in simplicity and applicability. However, the success of genetic algorithms in convergence to optimum is reported as better in this study. For further, one can apply other efficient metaheuristic methods to decrease the number of simulation runs that take a considerable amount of CPU time.

\section{Acknowledgement}

This research was supported by Çukurova University Scientific Research Project Commission.

\section{REFERENCES}

[1] Kershenbaum, A., Kermani, P., \& Grover, G. A. (1991). MENTOR: An algorithm for mesh network topological optimization and routing. In IEEE Transactions on Communications, 39(4), 503-513. https://doi.org/10.1109/26.81738

[2] Kumar, R. \& Banarjee, N. (2003). Multicriteria network design using evolutionary algorithm. Lecture Notes in Computer Science, 2724, 2179-2190. https://doi.org/10.1007/3-540-45110-2_113

[3] Khan, S. A. \& Engelbrecht, A. P. (2007). A new fuzzy operator and its application to topology design of distributed local area networks. Information Sciences, 177(13), 2692-2711. https://doi.org/10.1016/j.ins.2007.01.031

[4] Sem, K. \& Malhotra, S. (2008). Multi criteria network design using genetic algorithm. IET International Conference on Wireless, Mobile and Multimedia Networks, 56-60. https://doi.org/10.1049/cp:20080144

[5] Desai, J. \& Sen, S. (2010). A global optimization algorithm for reliable network design. European Journal of Operational Research, 200(1), 1-8. https://doi.org/10.1016/j.ejor.2008.12.016

[6] Cantor, D. G. \& Gerla, M. (1974). Optimal routing in a packetswitched computer network. IEEE Transactions on 
Computers, 23(10), 1062-1068. Retrieved from http://citeseerx.ist.psu.edu/viewdoc/download?doi=10.1.1.465 $.8084 \&$ rep $=$ rep $1 \&$ type $=$ pdf

[7] Frank, H. \& Chou, W. (1972). Topological optimization of computer networks. Proceedings of the IEEE, 60(11), 13851397. https://doi.org/10.1109/PROC.1972.8910

[8] Gavish, B. \& Hantler, S. (1983). An algorithm for optimal route selection in SNA networks. IEEE Transactions on Communications, 31(10), 1154-1161.

https://doi.org/10.1109/TCOM.1983.1095752

[9] Rajasekaran, S. \& Raghavachari, M. (1991). Optimal randomized algorithms for multipacket and wormhole routing on the mesh. University of Pennsylvania, Department of Computer \& Information Science Technical Reports, 1-14. Retrieved from https://repository.upenn.edu/cis_reports/329/

[10] Amiri, A. \& Pirkul, H. (1997). Routing and capacity assignment in backbone communication networks. Computers Ops Res., 24(3), 275-287.

https://doi.org/10.1016/S0305-0548(96)00049-4

[11] Amiri, A. \& Pirkul, H. (1999). Routing and capacity assignment in backbone communication networks under time varying traffic condition. European Journal of Operational Research, 117, 15-29. https://doi.org/10.1016/S0377-2217(98)00162-3

[12] Ferreira Filho, V. J. \& Galvao, R. D. (1994). A survey of computer network design problems. Investigacion Operativa, 4(3), 183-211.

[13] Cui, H., Li, J., Liu, X., \& Cai, Y. (2011). Particle swarm optimization for multi-constrained routing in telecommunication networks. I. J. Computer Network and Information Security, 4, 10-17. Retrieved from http://j.mecspress.net/ijcnis/ijcnis-v3-n4/IJCNIS-V3-N4-2.pdf

[14] Kawamato, H. \& Igarashi, A. (2012). Efficient packet routing strategy in complex networks. Physica A, 391, 895-904. https://doi.org/10.1016/j.physa.2011.08.064

[15] Seyed, J. M. \& Mohammad, T. (2007). FuzzyAntNet: a novel multi-agent routing algorithm for communications networks. Computer Sciences and Telecommunications, (1), 45-49. Retrieved from https://citeseerx.ist.psu.edu/viewdoc/ download?doi=10.1.1.129.6873\&rep=rep1\&type $=$ pdf

[16] Golbasi, H. (1996). A Path Constrained Approach to Dynamic Network Routing: İntegrating Virtual Clustering and Flow Deviation Algorithms. PhD Thesis, Lehigh University.

[17] Leung, K. \& Li, V. O. K. (2003). Flow assignment and packet scheduling for multipath routing. Journal of Communications and Networks, 5(3), 230-239. https://doi.org/10.1109/JCN.2003.6596817

[18] Tuba, M. (2009). Relation between static and dynamic optimization in computer network routing, Proceedings of the 8th WSEAS Int. Conf. on Artificial Intelligence, Knowledge Engineering \& Data Bases, 484-489. Retrieved from http://www.wseas.us/e-library/conferences/2009/cambridge/ AIKED/AIKED78.pdf

[19] Sykas, E. D. (1986). On the capacity assignment problem in packet-switching computer networks. Applied Mathematical Modelling, 10(5), 346-356. https://doi.org/10.1016/0307-904X(86)90094-6

[20] Ng, T. M. J. \& Hoang, D. B. (1987). Joint optimization of capacity and flow assignment in a packet-switched communications network. IEEE Transactions on Communications, 35(2), 202-209. https://doi.org/10.1109/TCOM.1987.1096743

[21] Neuman, I. (1989). Routing and capacity assignment in a network with different classes of messages. New York
University, Working Paper Series, 780-788. Retrieved from https://ssrn.com/abstract=1289684

[22] Wang, X., Li, D., Chen, H., Zhang, Y., Liu, H., \& Li, Y. (2020, June). A distributed optimization method to resource allocation problem on directed communication network under time delays. IEEE $4^{\text {th }}$ Information Technology, Networking, Electronic and Automation Control Conference, 1, 1335-1339. https://doi.org/10.1109//TNEC48623.2020.9084841

[23] Mahey, P., Benchakroun, A., \& Boyer, F. (2001). Capacity and flow assignment of data networks by generalized Benders decomposition. Journal of Global Optimization, 20(2), 169189. https://doi.org/10.1023/A:1011280023547

[24] Lin, X., Kwok, Y. \& Lau, V. K. N. (2003). A genetic algorithm based approach to route selection and capacity flow assignment. Computer Communications, 26, 961-974. https://doi.org/10.1016/S0140-3664(02)00240-2

[25] Queiroz, M. \& Humes Jr., C. (2003). A Heuristic for the continuous capacity and flow assignment. European Journal of Operational Research. 146, 444-459. https://doi.org/10.1016/S0377-2217(02)00219-9

[26] Batiha, K. (2015). Development of mathematical models to estimate optimum capacity assignment network connections. International Journal of Computer Applications, 129(16), 1214. https://doi.org/10.5120/ijca2015906964.

[27] Ran, C., Zhang, S., \& Huai, S. (2021). Link availability Prediction based on capacity optimization in MANET. Research Square. https://doi.org/10.21203/rs.3.rs-150048/v1

[28] Caiazza, C., Cicconetti, C., Luconi, V., \& Vecchio, A. (2021). Measurement-driven design and runtime optimization in edge computing: Methodology and tools. Computer Networks, 194, 108140. https://doi.org/10.1016/j.comnet.2021.108140

[29] Kojic, N., Neljin, R., \& Neljin, B. (2006). Neural network for optimization of routing in communication networks. Facta Universitatis (Nis) Ser.: Elec. Energ., 19(2), 327-329. https://doi.org/10.2298/FUEE0602317K

[30] Mukherjee, D. \& Acharyya, S. (2010). Ant colony optimization technique applied in network routing problem. International Journal of Computer Applications, 15(13), 6673. https://doi.org/10.5120/319-487

[31] Jalili, B. \& Dianati, M. (2010). Application of taboo search and genetic algorithms in planning and optimization of UMTS radio network. Proceedings of the 6th International Wireless Communications and Mobile Computing Conference, 143147. https://doi.org/10.1145/1815396.1815430

[32] Wu, J., Yue, W., \& Wang, S. (2006). Stochastic model and analysis for capacity optimization in communication networks. Computer Communications, 29, 2377-2385. https://doi.org/10.1016/j.comcom.2006.03.012

[33] Lin, Y-K. \& Huang, C. F. (2013). System reliability with routing scheme for a stochastic computer network under accuracy rate. International Journal of Industrial Engineering, 20(5-6), 319-328.

[34] Banks, J., Carson, J. S., Nelson, B. L. \& Nicol, D. M. (2000). Discrete Event Systems Simulation, (3rd Ed.). New Jersey: Pearson, (Chapter 12).

[35] Tekin, E. \& Sabuncuoğlu, H. (2004). Simulation optimization: A comprehensive review on theory and applications. IIE Transactions, 36, 1067-1081. https://doi.org/10.1080/07408170490500654

[36] Shen, J., Xu, F. \& Zheng, P. (2005). A tabu search algorithm for the routing and capacity assignment problem in computer networks. Computers and Operations Research, 32, 27852800. https://doi.org/10.1016/j.cor.2004.04.004

[37] Geem, Z. W., Kim, J. H. \& Loganathan G. V. (2001). A new heuristic optimization algorithm: harmony search. Simulation, 76-2, 60-68. https://doi.org/10.1177\%2F003754970107600201 
[38] Koza, J. R. (1992). Genetic Programming: On the Programming of Computers by Means of Natural Selection. Cambridge, MA: the MIT Press.

[39] Yadav, P., Kulmar, R., Panda, S. K., \& Chang, C. S. (2012). An intelligent tuned harmony search algorithm for optimisation. Information Sciences, 196, 47-72. https://doi.org/10.1016/j.ins.2011.12.035

\section{Authors' contacts:}

Nuşin Uncu, Assistant Prof. Dr.

(Corresponding author)

Adana Alparslan Türkeş Science and Technology University,

Department of Industrial Engineering,

Balcalı Mahallesi, Çatalan Caddesi No: 201/1,

01250 Sarıçam-Adana/Turkey

nuncu@atu.edu.tr

Rızvan Erol, Prof. Dr.

Çukurova University,

Department of Industrial Engineering,

Balcalı Kampüsü,

Sarıçam-Adana/Turkey

rerol@cu.edu.tr 